\title{
MARTINGALE-DIFFERENCE GIBBS RANDOM FIELDS AND CENTRAL LIMIT THEOREM
}

\author{
B.S. Nahapetian and A.N. Petrosian \\ Armenian Academy of Sciences, Institute of Mathematics \\ Marshal Bagramian Ave. 24-B, 375019 Yerevan, Armenia
}

\begin{abstract}
The notion of a martingale difference random field is introduced, and sufficient conditions for a Gibbs random field to possess the martingale difference property are studied. Various central limit theorems for such random fields are given.
\end{abstract}

Let $\mathbf{Z}^{\nu}$ be a lattice $(\nu \geq 1)$ and $W$ be the family of all its finite subsets.

Definition 1. We say that a random field $\xi_{t}, t \in \mathbf{Z}^{\nu}$, is a martingaledifference random field with respect to a given sequence of increasing finite subsets (s.i.f.s.) $V_{i} \in W, i=1,2 \ldots$, if $\mathbf{E}\left|\xi_{t}\right|<\infty, t \in \mathbf{Z}^{\nu}$, and

$$
\mathbf{E}\left(\xi_{t} \mid \xi_{s}, s \in V_{i-1}\right)=0, \quad \text { a.s. }
$$

for all $t \in V_{i} \backslash V_{i-1}$ and $i=2,3, \ldots$.

If (1) holds for all s.i.f.s. then the random field is called simply a martingaledifference random field.

Definition 2. We say that a random process $S_{V}, V \in W$, forms a martingale with respect to a s.i.f.s. $V_{i}, i=1,2, \ldots$, if $\mathbf{E}\left|S_{V}\right|<\infty, V \in W$, and the relation

$$
\mathbf{E}\left(S_{V_{i}} \mid S_{V_{1}}, S_{V_{2}}, \ldots, S_{V_{i-1}}\right)=S_{V_{i-1}}, \quad \text { a.s. }
$$

is valid for all $i=2,3, \ldots$.

It is easy to see that if a random field $\xi_{t}, t \in \mathbf{Z}^{\nu}$, is a martingale-difference with respect to a s.i.f.s. $V_{i}, i=1,2, \ldots$, then the random process $S_{V}=\sum_{t \in V} \xi_{t}$, $V \in W$, is a martingale with respect to $V_{i}, i=1,2, \ldots$. Conversely, if a random process $S_{V}=\sum_{t \in V} \xi_{t}, V \in W$, is a martingale with respect to any s.i.f.s. then the random field $\xi_{t}, t \in \mathbf{Z}^{\nu}$, is a martingale-difference random field.

There is a wide spectrum of examples of random fields and processes satisfying the conditions in these definitions.

Example 1. Let $V_{i}, i=1,2, \ldots$, be a s.i.f.s. and $\Delta_{j}=V_{j} \backslash V_{j-1}, j=2,3, \ldots$ Suppose a random field $\xi_{t}, t \in \mathbf{Z}^{\nu}$ has the properties: $\mathbf{E} \xi_{t}=0, t \in \mathbf{Z}^{\nu}$, and $\xi_{t}$, $\xi_{s}$ are independent for $t \in \Delta_{j}, s \in \Delta_{k}, j \neq k$. Then it is a martingale-difference with respect to $V_{i}, i=1,2, \ldots$. 
Example 2. Let $\xi_{t}, t \in \mathbf{Z}^{\nu}$, be a random field taking values in a separable complete metric space $X$ with a $\sigma$-finite measure $\mu, \mu(X)>c$, defined on its Borel subsets. Suppose the finite dimensional distributions of this random field are absolutely continuous with respect to the product measures $\mu_{v}=\mu^{|v|}, v \in W$ $(|\cdot|$ stands for the number of points in a finite set) and suppose the densities $p_{V}\left(x_{t}, t \in V\right), V \in W$, are strictly positive. Moreover, suppose $q_{V}\left(x_{t}, t \in V\right)$, $V \in W$, is another system of consistent densities. Then the process

$$
S_{V}=\frac{q_{V}\left(\xi_{t}, t \in V\right)}{p_{V}\left(\xi_{t}, t \in V\right)}, \quad V \in W
$$

is a martingale with respect to any s.i.f.s.

Example 3. Suppose $\xi_{t}, t \in \mathbf{Z}^{\nu}$, is a random field satisfying $\mathbf{E}\left|\xi_{t}\right|<\infty$, $t \in \mathbf{Z}^{\nu}$, and

$$
\mathbf{E}\left(\xi_{t} \mid \xi_{s}, s \in \mathbf{Z}^{\nu} \backslash\{t\}\right)=0 \quad \text { a.s. }
$$

Then $\xi_{t}, t \in \mathbf{Z}^{\nu}$, is a martingale-difference random field.

Example 4. Suppose $\mathbf{Z}^{\nu}=\cup_{j} T_{j}, T_{j} \cap T_{k}=\emptyset, j \neq k$; and suppose $\xi_{t}$, $t \in \mathbf{Z}^{\nu}$, is a random field having the property: $S_{V}=\sum_{t \in V} \xi_{t}, V \subset T_{j}$, is a martingale with respect to any s.i.f.s. $V_{i}, i=1,2, \ldots, V_{i} \subset T_{j}$ for any fixed $j=1,2, \ldots$ If $S_{V}$ and $S_{\tilde{V}}$ are, in addition, independent for $V \subset T_{j}$ and $\tilde{V} \subset T_{k}$, $j \neq k$, then the random field $\xi_{t}, t \in \mathbf{Z}^{\nu}$, is a martingale-difference.

The following example is a special case of Example 4.

Example 5. Suppose a random field $\xi_{t}, t \in \mathbf{Z}^{2}, \mathbf{E}\left|\xi_{t}\right|<\infty, t \in \mathbf{Z}^{2}$ has the property: for any $p, k \in \mathbf{Z}^{1}$

$$
\mathbf{E}\left(\xi_{(p, k)} \mid \ldots \xi_{(p-1, k)}, \xi_{(p+1, k)} \ldots\right)=0 \quad \text { a.s. }
$$

and $\xi_{(p, k)}, \xi_{(q, j)}$ are independent for $k \neq j$. Then the random field $\xi_{t}, t \in \mathbf{Z}^{2}$, is a martingale-difference.

Note that each of the examples considered here has an analogy in the theory of martingales.

We introduce next a new construction of martingale-difference random fields which is useful also in the theory of Gibbs random fields.

Suppose $Y, Y \subset R^{1}$, is a symmetric set with respect to the origin (i.e., if $y \in Y$ then $-y \in Y$ ) and $\mathscr{B}(Y)$ is the $\sigma$-algebra of its Borel subsets. Consider a symmetric measure $\mu$ on $\mathscr{B}(Y)$ (i.e., $\mu(B)=\mu(-B), B \in \mathscr{B}(Y))$ satisfying

$$
\int_{Y}|y| \mu(d y)<\infty
$$

Lemma 1. Let $\xi_{t}, t \in \mathbf{Z}^{\nu}$, be a random field taking values in $Y$. Suppose its finite-dimensional distributions are absolutely continuous with respect to the 
product-measures $\mu^{|v|}, V \in W$, with densities $p_{V}\left(y_{t_{1}}, y_{t_{2}}, \ldots, y_{t_{|V|}}\right), V \in W$, satisfying

$$
p_{V}\left(\theta_{1} y_{t_{1}}, \theta_{2} y_{t_{2}}, \ldots, \theta_{t_{|V|}} y_{t_{|V|}}\right)=p_{V}\left(y_{t_{1}}, y_{t_{2}}, \ldots, y_{t_{|V|}}\right), \quad V \in W
$$

for any $\theta_{i} \in\{-1,1\}, i=1,2, \ldots,|V|$ (the superparity property). Then the random field $\xi_{t}, t \in \mathbf{Z}^{\nu}$, is a martingale-difference.

Proof. It is sufficient to show that $S_{V}=\sum_{t \in V} \xi_{t}, V \in W$, is a martingale with respect to an arbitrary s.i.f.s. That is, for any sequence $V_{i} \in W, V_{i} \subset V_{i+1}$, $i=1,2, \ldots$, one has

$$
\sum_{t \in V_{i} \backslash V_{i-1}} \mathbf{E}\left(\xi_{t} \mid S_{V_{1}}, S_{V_{2}}, \ldots, S_{V_{i-1}}\right)=0 \quad \text { a.s. }
$$

or

$$
\sum_{t \in V_{i} \backslash V_{i-1}} \int_{A} \xi_{t} \mathbf{P}(d \omega)=0 \quad \text { for any } A \in \sigma\left(\xi_{s}, s \in V_{i-1}\right) .
$$

This relation can be rewritten in the following form: for any

$$
\begin{gathered}
B \in \mathscr{B}\left(Y_{V_{i-1}}\right), \quad V_{i-1} \in W, \\
\sum_{t \in V_{i} \backslash V_{i-1}} \int_{B \times Y_{t}} x p_{V_{i-1} \cup\{t\}}(y, x) \mu_{t}(d x) \mu_{V_{i-1}}(d y)=0 .
\end{gathered}
$$

However, taking into account the superparity of the densities $p_{V}$ and the symmetry of the measure $\mu_{t}$ we have

$$
\int_{Y_{t}} x p_{V_{i-1} \cup\{t\}}(y, x) \mu_{t}(d x)=0 .
$$

The lemma is proven.

Now we introduce the notion of a Gibbs random field. Note that the definition given below is not general.

Let $\left(Y_{t}, \mathscr{B}_{t}, \mu_{t}\right), t \in \mathbf{Z}^{\nu}$, be a copy of $(Y, \mathscr{B}, \mu)$. A system of measurable functions $\Phi=\left\{\Phi_{V}, V \in W\right\}$, defined on $\left(Y_{V}, \mathscr{B}_{V}, \mu_{V}\right)$, is called a potential. Here

$$
Y_{V}=\otimes_{t \in V} Y_{t}, \quad \mathscr{B}_{V}=\otimes_{t \in V} \mathscr{B}_{t}, \quad \mu_{V}=\otimes_{t \in V} \mu_{t},
$$

( $\otimes$ is here also the symbol of the Cartesian product).

For any $v \in W$ and $\bar{y} \in Y_{\mathbf{Z}^{\nu} \backslash V}$ we define a function

$$
U_{V}^{\bar{y}}(y)=\sum_{J \subset V} \sum_{\tilde{J} \subset \mathbf{Z}^{\nu} \backslash V} \Phi_{J \cup \tilde{J}}\left(y_{J}, \bar{y}_{\tilde{J}}\right), y \in Y_{V}, \quad y_{J}=\left(y_{s}, s \in J\right), \bar{y}_{\tilde{J}}=\left(\bar{y}_{s}, s \in \tilde{J}\right),
$$


which is called the potential energy. This quantity is finite if for example the condition

$$
\sup _{a \in \mathbf{Z}^{\nu}} \sum_{J: a \in J \in W} \sup _{y \in Y_{J}}\left|\Phi_{J}(y)\right|<\infty
$$

is satisfied.

Define

$$
\left(q_{V}^{\bar{y}}\right)_{I}(y)=\frac{\int_{Y_{V \backslash I}} \exp \left\{-U_{V}^{\bar{y}}(y, z)\right\} \mu_{V \backslash I}(d z)}{\int_{Y_{V}} \exp \left\{-U_{V}^{\bar{y}}(z)\right\} \mu_{V}(d z)}, \quad y \in Y_{I}, \bar{y} \in Y_{\mathbf{Z}^{\nu} \backslash V} .
$$

Suppose that for any $I \in W$ there exist an increasing sequence $V_{k} \in W, k=$ $1,2, \ldots$, satisfying $\cup_{k} V_{k}=\mathbf{Z}^{\nu}$, and boundary conditions $\bar{y}_{k} \in Y_{\mathbf{Z}^{\nu} \backslash V_{k}}$ such that the limes (uniform with respect to $y \in Y_{I}$ )

$$
\lim _{k \rightarrow \infty}\left(q_{V_{k}}^{\bar{y}_{k}}\right)_{I}(y)=p_{I}(y)
$$

exists. Then the system of finite-dimensional densities $\left\{p_{I}, I \in W\right\}$ is consistent, and hence there exists a random field called a Gibbs random field.

Definition 3. We say that a potential $\Phi$ is a superparity potential if for any $y=\left(y_{t_{1}}, \ldots, y_{t_{|V|}}\right), y_{t_{i}} \in Y, V \in W$, the relation

$$
\Phi_{V}\left(\theta_{1} y_{t_{1}}, \ldots, \theta_{|V|} y_{t_{|V|}}\right)=\Phi\left(y_{t_{1}}, \ldots, y_{t_{|V|}}\right), \quad \theta_{i} \in\{-1,1\},
$$

holds.

Lemma 2. Let $\Phi$ be a potential satisfying

$$
\sup _{a \in \mathbf{Z}^{\nu}} \sum_{J: a \in J \in W} \sup _{y \in Y_{J}}\left|\Phi_{J}(y)\right|<\infty .
$$

If the potential $\Phi$ has the superparity property, then the corresponding Gibbs random field is a martingale-difference.

This lemma follows from the superparity of the potential $\Phi$ combined with Lemma 1.

One can construct many examples of superparity potentials $\Phi=\left\{\Phi_{V}, V \in\right.$ $W\}$. For example, for $V \in W$ define

$$
\Phi_{V}\left(y_{t}, t \in V\right)= \begin{cases}\prod_{t \in V}\left|y_{t}\right|(\operatorname{Diam} V)^{-\gamma}, & \gamma>0,|V| \leq 2 \\ 0, & |V|>2\end{cases}
$$

or

$$
\Phi_{V}\left(y_{t}, t \in V\right)=\exp \left\{-\sup _{t \in V}\left|y_{t}\right||V|^{\gamma}\right\}, \quad \gamma>0 .
$$

There are several well-known articles dealing with the central limit theorem for martingale-difference random processes.

The next theorem is a direct consequence of the results of Brown [3] and Dvoretsky [5]. 
Theorem 1. Let $S_{V}, V \in W$, be a martingale asociated with a s.i.f.s. $V_{i}$, $i=1,2, \ldots$. Suppose the following conditions hold:

1) $\mathbf{D} \eta_{\Delta_{j}} \geq \sigma^{2}\left|\Delta_{j}\right|, \sigma^{2}>0, \mathbf{E}\left|\eta_{\Delta_{j}}\right|^{2+\delta} \leq C\left|\Delta_{j}\right|^{1+\delta / 2}, 0<C<\infty, \delta>0$; here $\mathbf{D}$ stands for the variance, and $\bar{\Delta}_{j}=V_{j} \backslash V_{j-1}, \eta_{\Delta_{j}}=S_{V_{j}}-S_{V_{j-1}}$, $j=2,3, \ldots$;

2) $\lim _{n \rightarrow \infty}\left(\mathbf{D} S_{V_{n}}\right)^{-1} \sum_{j=2}^{n} \operatorname{Cov}\left(\eta_{\Delta_{j}}^{2}, \operatorname{sgn} I_{j}\right)=0$, where

$I_{j}=\mathbf{E}\left(\eta_{\Delta_{j}}^{2} \mid \eta_{\Delta_{1}}, \ldots, \eta_{\Delta_{j-1}}\right)-\mathbf{E} \eta_{\Delta_{j}}^{2}, j=2,3, \ldots$

Then for any $x \in R^{1}$

$$
\lim _{n \rightarrow \infty} \mathbf{P}\left(\left(\mathbf{D} S_{V_{n}}\right)^{-1 / 2}\left(S_{V_{n}}-\mathbf{E} S_{V_{n}}\right)<x\right)=\frac{1}{\sqrt{2 \pi}} \int_{-\infty}^{x} e^{-u^{2} / 2} d u,
$$

i.e., the central limit theorem holds.

The conditions of this type can be checked for the martingales $S_{V}, V \in W$, which are sums of weakly dependent random variables (see [1], [6-8]). In this paper we restrict ourselves to the consideration of martingales which are sums of components of a weakly dependent random field. We use the following mixing coefficients

$$
\begin{aligned}
& \alpha_{m, n}(p)=\sup _{m, n \in \mathrm{N} \cup\{\infty\}}\left\{\alpha\left(M_{I}, M_{V}\right) ; I, V \in W, \varrho(I, V) \geq p,|I| \leq m,|V| \leq n\right\}, \\
& \alpha\left(M_{I}, M_{V}\right)=\sup _{A \in M_{I}, B \in M_{V}}|P(A B)-P(A) P(B)|, \\
& M_{I}=\sigma\left(\xi_{t}, t \in I\right), \quad M_{V}=\sigma\left(\xi_{t}, t \in V\right), \\
& \varrho(I, V)=\inf _{s \in V, t \in I} \varrho(s, t), \quad \varrho(s, t)=\max _{1 \leq i \leq \nu}\left|s^{(i)}-t^{(i)}\right|, \quad s, t \in \mathbf{Z}^{\nu} .
\end{aligned}
$$

Theorem 2. Let $\xi_{t}, t \in \mathbf{Z}^{\nu}$, be a martingale-difference field satisfying $\mathbf{E} \xi_{t}=0, \inf _{t \in \mathbf{Z}^{\nu}} \mathbf{E} \xi_{t}^{2}=\sigma_{0}^{2}>0$, and

$$
\left|\xi_{t}\right|<C, \quad t \in \mathbf{Z}^{\nu}, \quad \text { a.s. }
$$

Suppose

$$
\alpha_{m, n}(p) \leq f(m) \alpha(p)
$$

where $f(m)$ is some function and $\alpha(p) \rightarrow 0$ as $p \rightarrow \infty$. Then for any increasing sequence of cubes $V_{n} \subset \mathbf{Z}^{\nu}, n=1,2, \ldots$

$$
\lim _{n \rightarrow \infty} \mathbf{P}\left(\left(\mathbf{D} \sum_{t \in V_{n}} \xi_{t}\right)^{-1 / 2} \sum_{t \in V_{n}} \xi_{t}<x\right)=\frac{1}{\sqrt{2 \pi}} \int_{-\infty}^{x} e^{-u^{2} / 2} d u .
$$

Note that in contrast to the well-known central limit theorems for weakly dependent random fields (see for example [2], [4], [9-11]) the mixing coefficients in Theorem 2 do not depend on the dimension of the lattice $\mathbf{Z}^{\nu}$. 
Theorem 3. Let $\Phi$ be a translation invariant potential having the superparity property. Suppose $\Phi$ has a sufficiently small norm

$$
\|\Phi\|=\sum_{J: 0 \in J \in W}|J| \sup _{y \in Y_{J}}\left|\Phi_{J}(y)\right|
$$

Then the central limit theorem holds for the corresponding Gibbs random field for any increasing sequence of cubes $V_{n} \subset \mathbf{Z}^{\nu}, n=1,2, \ldots$

This theorem is a consequence of Theorem 2 and Theorem 9.1.1 of [10].

\section{References}

[1] Billingsley, P.: The Lindeberg-Levy theorem for martingales. - Proc. Amer. Math. Soc. $12,1961,788-792$.

[2] Bolthausen, E.: On the central limit theorem for stationary mixing random fields. Ann. Probab. 10, 1982, 1049-1052.

[3] Brown, B.M.: Martingale central limit theorems. - Ann. Math. Statist. 42, 1971, 59-66.

[4] Bulinski, A.V.: The central limit theorem and invariance principle for mixing random fields. - The First World Congress of the Bernoulli Society, Abstracts, Vol. 2, Tashkent, 1986, 640 .

[5] Dvoretsky, A.: Asymptotic normality for sums of dependent random variables. - Proceedings of the Sixth Berkeley Symposium of Mathematical Statistics. University of California Press, Berkeley, 1972, 513-535.

[6] Gordin, M.I.: The central limit theorem for the stationary processes. - Soviet Math. Dokl. 10, 1969, 1174-1176 (English translation of Dokl. Akad. Nauk SSSR 188, 1969, 739-741).

[7] Ibragimov, I.A.: A central limit theorem for a class of dependent random variables. Theory Probab. Appl. 8, 1963, 83-84 (English translation of Teor. Veroyatnost. i Primenen. 8, 1963, 89-94).

[8] Liptser, P.S., and A.N. Shiryayev: Theory of martingales. - Nauka, Moscow, 1986 (Russian).

[9] Nahapetian, B.S.: An approach to proving limit theorems for dependent random variables. - Theory Probab. Appl. 32, 1987, 535-539 (English translation of Teor. Veroyatnost. i Primenen. 32, 1987, 589-594).

[10] Nahapetian, B.S.: Limit theorems and some applications in statistical physics. Teubner-Texte zur Mathematik, Leipzig, 1990.

[11] Takahata, H.: On the central limit theorem for weakly dependent random fields. - Yokohama Math. J. 31, 1983, 67-77. 